Ann. Biol. anim., Bioch., Biophys., I965, 5 (4), 4rg-443.

\title{
RELATIONS ENTRE LES CARACTÉRISTIQUES DU SPERME ET LA FERTILITÉ
}

\author{
C. Van DUIJN jr. \\ Département de Biophysique, \\ Institut de Recherches zootechniques "Schoonoord ". Zeist, (Pays-Bas)
}

\section{SOMMAIRE}

Une équation générale de fertilité est proposée. Elle est déduite de considérations cinétiques simples exprimant la probabilité de fécondation d'un éjaculat en fonction du nombre, de la vitesse moyenne, de la période de demi-valeur et de la fécondance relative des spermatozoïdes, ainsi que des paramètres de la population femelle. De plus, il est indiqué comment ces données dépendent des caractéristiques biochimiques et des anomalies de l'acrosome.

La vérification de l'équation de fertilité et des expressions qui en sont déduites pour l'apprécia. tion du sperme dans la pratique, a donné une concordance complète entre les prévisions théoriques et les résultats pratiques sur des données provenant de Bovins, Porcins et Volailles.

Enfin, l'auteur expose la méthode de détermination simple des caractéristiques du sperme requises dans la pratique.

\section{INTRODUCTION}

I a relation entre les caractéristiques du sperme et la fertilité est d'une importance fondamentale pour la biologie de la reproduction en général; elle présente un intérêt économique direct dans la pratique de l'insémination artificielle, car une appréciation aussi correcte que possible de la fécondance du sperme à utiliser est en partie décisive pour la rentabilité.

Le vaste matériel recueilli par de nombreuses personnes depuis la mise en pratique de l'insémination artificielle n'a jamais été, en général, soumis qu'à des méthodes statistiques simples, sans toujours vérifier s'il était correct ou non d'appliquer certaines techniques mathématiques élémentaires.

Il est devenu nécessaire de mesurer avec précision les caractéristiques de la motilité des spermatozoïdes qui se trouve dans la plupart des cas estimée à vue 
d'wil d'une manière plus ou moins subjective, ce qui comporte de gros risques d'erreurs, car toutes sortes d'effets secondaires peuvent intervenir.

Or, il est devenu possible d'obtenir des informations précises sur la vitesse moyenne $v$ des spermatozoïdes contenus dans un éjaculat et qui se meuvent normalement, sur la distribution de leur vitesse $f\left(v^{\prime}\right)$, sur leur nombre et leur concentration par la méthode photo-électrique développée en I957, par RIKMENSPori, à 1'Institut de Recherches zootechniques "Schoonoord ".

Le dilueur utilisé contient I5 p. Ioo de jaune d'ueuf et 85 p. Ioo d'une solution de citrate de sodium; elle est clarifiée par ultra-centrifugation suivie de deux ultrafiltrations.

On place sous un microscope avec éclairage à fond noir du sperme dilué dans du citrate-jaune d'œuf. L'image est projetée sur un diaphragne du format de la tête d'un spermatozoïde. Au moyen d'une lentille auxiliaire, ce diaphragme se trouve à son tour reproduit sur la cathode d'un photomultiplicateur, lequel, sitôt qu'un objet diffusant la lumière passe à l'endroit correspondant du champ, reçoit un signal lumineux. Celui-ci, produit, une émission des électrons qui, par appareillage électronique auxiliaire, donnent un signal lisible sur la bande d'un appareil enregistreur.

Les enregistrements permettent ensuite de déterminer le nombre des spermatozoïdes et leurs vitesses respectives.

L'analyse des données rassemblées au cours de plusieurs années avec cette méthode, a montré, contre toute attente, une relation significative entre la vitesse initiale des spermatozoïdes et la rapidité de sa diminution (VAx 1)viJN, rg62). Cette constatation a mené à la conclusion que, une grande vitesse initiale des spermatozoïdes ne peut pas être considérée comme une caractéristique favorable en soi, puisqu'elle va de pair avec une chance accrue de diminution rapide, tant de cette vitesse que du nombre des spermatozoïdes se déplaçant encore normalement.

'On a constaté que les caractéristiques de vieillissement du sperme, à la diminution de la vitesse avec le temps ( - ov/ot) et le temps nécessaire à la diminution de moitié du nombre des spermatozoïdes mobiles (période de demi-valeur) $t_{1 / 2}(\mathrm{~N})$, sont plus importantes que la vitesse moyenne $\bar{v}$. Cette conclusion fut encore confirmée, lorsqu'il s'est avéré que lors des mois accusant les pourcentages de fécondation les plus élevés chez les bovins, les vitesses moyennes des spermatozoïdes étaient plus basses, alors que les concentrations de ceux qui se meuvent normalement, et leurs paramètres de survie possédaient les valeurs les plus favorables ; ces rapports étaient opposés lors des mois pendant lesquels les pourcentages de fécondation étaient les plus bas.

Cependant, il apparut bientôt que les limites de la recherche empirique avec analyse de corrélation étaient atteintes.

Une relation rationnelle entre les différents paramètres ne peut être obtenue par une simple élaboration statistique des résultats d'observation. C'est pourquoi nous sommes passé à une approche fondamentale théorique du problème de la fertilité, en vérifiant la validité de notre équation sur le vaste matériel expérimental rapporté dans la littérature scientifique d'une part, et les données spécifiques recueillies au cours de plusieurs années à l'Institut d'autre part.

Cette méthode a conduit à la mise au point d'une théorie cinétique des chances de fécondation qui comprend, par principe, toutes les caractéristiques de fécondation imaginables et qui s'appuie sur tun minimum d'hypothèses dont aucune n'est liée 
spécifiquement à la physiologie de la reproduction d'un animal déterminé, si bien que les résultats sont d'une validité très générale. Sur la base de cette théorie avec laquelle toutes les données expérimentales analysées paraissent en concordance, il est possible d'indiquer de meilleures méthodes d'appréciation du sperme et de calculer des estimations de fécondité exactes, en utilisant diverses types de caractéristiques du sperme.

\section{I. - LA THÉORIE CINÉTIQUE}

Les points de départ fondamentaux de la théorie se résument comme suit :

$\mathrm{I}^{0}$ Le nombre des spermatozoïdes $\mathrm{N}$ capables de féconder et la rapidité de déplacement $\mathrm{N} \cdot \bar{v}$ $(\bar{y}=$ vitesse moyenne des spermatozoïdes dans des conditions normalisées) décroissent exponentiellement avec le temps, donc:

$$
-\frac{d \mathrm{~N}}{d t}=k_{\mathrm{N}} \cdot \mathrm{N}(\mathrm{I} a) \quad \text { et } \quad-\frac{d \mathrm{~N} \cdot \bar{v}}{d t}=k_{\mathrm{N} v} \cdot \mathrm{N} \cdot \bar{v}
$$

Cette hypothèse est fondée sur un matériel expérimental important rassemblé au cours des io dernières années (Rikmenspolit, i957, ig6o; Rikmenspoel et Van Duijn, ig6o; Van Duijn et Rikmenspoel, ig6o; Van Duijn, i 1963 ).

$2^{0}$ Si les spermatozoïdes s'approchent d'un ovule en nombre constant par unité de temps $\delta t$, sans que des effets de détérioration ou de mortalité puissent être supposés lors de la période de transport dans les voies $q$ l'accroissement de la chance de fécondation avec le temps pendant lequel l'apport a lieu, doit se calculer comme suit :

$$
\frac{d \mathrm{~F}}{d t}-k_{\mathrm{F}}(\mathrm{F} \infty-\mathrm{F})
$$

dans laquelle la formule $\mathrm{F}_{\infty}$ représente la chance de fécondation maximum dans la population donnée et $F$ la chance que la fécondation soit obtenue au temps $t$ dans les conditions données.

Étant donné qu'en réalité ni le nombre des spermatozoïdes fécondés, ni leur vitesse ne demeurent constants, la relation réelle ne pourra être obtenue qu'en tenant compte du rythme de diminution indiqué en $\mathrm{x} b$. Ceci amène à l'équation différentielle :

$$
\frac{d \mathrm{~F}}{\bar{d} \mathrm{~N} \bar{v}}=\frac{k_{\mathrm{F}} \cdot\left(\mathrm{F}_{\infty}-\mathrm{F}\right)}{k_{\mathrm{N} v} \cdot \mathrm{X} \cdot \ddot{y}}
$$

Si la vitesse moyenne des spermatozoïdes est invariable, ce qui est le cas lorsque l'insémination s'effectue avec des doses diverses du même éjaculat, ou si la vitesse n'a aucune influence sur les chances de fécondation, l'équation (3) se réduit en :

$$
\frac{d \mathrm{~F}}{d \overline{\mathrm{N}}}=\frac{k_{\mathrm{F}}\left(\mathrm{F}_{\infty}-\mathrm{F}\right)}{k_{\mathrm{N}} \cdot \mathrm{N}}
$$

Les équations (3) et (4) peuvent être intégrées; dans les conditions limites $\mathrm{N}=\mathrm{N}_{i}, \mathrm{~N} \bar{v}=(\mathrm{N} \bar{v})_{i}$ pour $\mathrm{F}=0 ; \mathrm{N}_{i}$ et $(\mathrm{N} \bar{v})_{i}$ sont des valeurs seuil qui doivent être dépassées pour amener la chance de fécondation à un taux supérieur à zéro. Aucune hypothèse spécifique n'est formulée pour l'existence d'une valeur seuil, mais celle-ci est une exigence mathématique, puisque, pour réaliser une fécondation, il faut au moins un spermatozoïde fertile.

Toutefois, le début de la fonction ne peut pas être fixé à I, étant donné que tous les spermatozoïdes mobiles d'un éjaculat ne sont pas nécessairement fertiles. L'intégration de (3) donne l'équation de fertilité générale

$$
\mathrm{F}=\mathrm{F}_{\infty}\left(\mathrm{I}-\exp \left[-\frac{k_{\mathrm{F}}}{k_{\mathrm{N} v}} \cdot \ln \frac{(\mathrm{N} \bar{\nu})_{o}}{(\mathrm{~N} \bar{\nu})_{i}}\right]\right)
$$

formule dans laquelle $(\mathrm{N} \bar{v})_{0}$ représente alors la valeur de $\mathrm{N} \bar{v}$ au moment même de l'insémination (naturelle ou artificielle). 
Lorsque $\bar{v}$ est invariable, la relation restreinte de (4) est valable, donnant à l'intégrale une forme correspondante à celle de (5) avec $\mathrm{N}$ au lieu de $\mathrm{N}$.

L'infuence des femelles sur les chances de fécondation se traduit dans les valeurs numériques pour $\mathbf{F}_{\infty}$ et pour $k_{\mathrm{F}}$.

Toutes les propriétés imaginables des spermatozoïdes qui - tout en étant indépendantes des caractéristiques de motilité et de la durée de survie - pourraient encore exercer leur influence sur la fertilité, sont comprises dans la valeur numérique de $\mathrm{N}_{i}$. Si, par exemple, un éjaculat contient $5 \circ$ p. 100 de spermatozoïdes avec une anomalie qui empêche la fécondation, $\mathrm{N}_{i}$ devient deux fois plus grand que lorsque tous les spermatozoïdes sont normaux.

D'autre part, la valeur $N_{i}$ (tout comme celles de $\mathrm{F}$ et de $\mathrm{F}_{x_{x}}$ ) sera aussi fonction du système employé pour exprimer la fertilité, si cette denière est exprimée sous la forme d'un pourcentage de gestation (ou une quantité s'y rapportant comme le pourcentage des "non-retours »), $\mathrm{N}_{i}$ sera toujours plus élevé que dans le cas de l'observation directe de la pénétration d'un spermatozoïde dans l'wuf.

Bien que celle-ci soit théoriquement la caractéristique essentielle, elle s'avère peu maniable et donc peu intéressante pour la pratique, car la cause de cette différence est que la pénétration d'un spermatozoïde dans l'ovule est une condition indispensable, mais non suffisante pour le développement de l'ovule en embryon.

\section{II. - TRANSFORMATION DE L','ÉQUATION DE FERTILITÉ ET VÉRIFICATION}

L'équation de fertilité peut être transformée en une relation rectiligne par conversion en logarithme $\left(\mathrm{F}_{\infty} /\left(\mathrm{F}_{\infty}-\mathrm{F}\right)=f[\log (\mathrm{N} \bar{v})]\right.$ ou $f[\log (\mathrm{N})]$ (voir fig. 3-5). La pente de la droite représente la valeur de $k_{\mathrm{F}} / k_{\mathrm{N}} \vec{v}$, soit $k_{\mathrm{F}} / k_{\mathrm{x}}$, alors que la valeur seuil $(\mathrm{N} v)_{i}$, soit $N_{i}$ se trouve au point d'intersection de la droite avec l'abscisse. La valeur de $F_{\infty}$ nécessaire pour pouvoir opérer la transformation peut être déterminée par estimations itératives, d'une série d'observations, ou d'une façon plus rapide et plus simple en appliquant la méthode graphique de HEYDECKER (I925) (voir fig. 6).

Les transformations en droites sont toujours nécessaires afin de pouvoir pratiquer l'analyse de la corrélation et de la régression linéaire. Sinon, seules peuvent être appliquées les méthodes statistiques non paramétriques car aucun des paramètres ne répond aux conditions qui sont valables pour l'application des statistiques normales (voir III).

Après transformation linéaire, la concordance entre la théorie et les résultats expérimentaux peut être mesurée par le coefficient de corrélation linéaire $r$.

Il fut calculé sur des données publiées par WIILETT et LARSOx (I952) : en supposant la vitesse moyenne constante on obtient $r=0,98, P \leqslant \mathbf{I O}^{-6}$ (fig. I et 2 ) 72336 inséminations de 45 r éjaculats de Taureaux. Pour le matériel recueilli sur des volailles, TANEJA et Gowe (I962) en supposant également une vitesse moyenne invariable, le résultat était de $: r=0,96, \mathrm{P}<\mathrm{IO}^{-8}$ (voir fig. 3 et 4). Le matériel rassemblé sur les porcins se réduit à peu de chose ; les données de HANCOCK et HoVEı, (I G6I) qui avaient déterminé les résultats de fécondation en examinant la division de l'œuf après l'abattage des 'Truies inséminées, pouvaient de justesse servir à la mise au point d'une analyse avec comme résultat $r=0,8 \mathrm{I}, 0,05$ $>\mathrm{P}>0,02$.

Dans les cas de motilité variable les données fournies par la littérature sont également en accord avec la théorie. BRAN'TON, KELLGREN et PATRICK (I953) ont publié des indices sur les résultats de fécondation et sur le produit du nombre total de spermatozoïdes avec des indices de motilité estimés à vue d'œil $m$, basés sur 


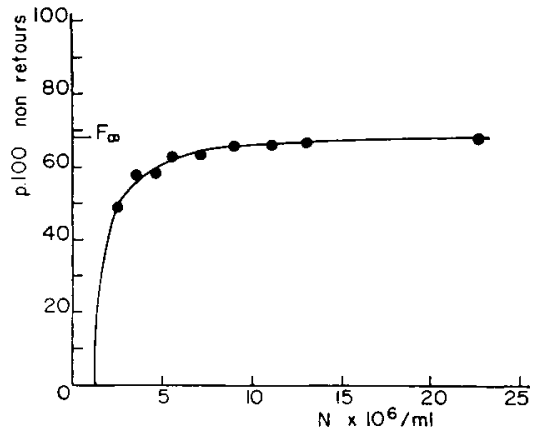

FIG. 1. - Relation entre le nombre des spermatozoides et les résultals de fécondation, calculée d'après les données expérimenlales de Willett et LARSON (1952)

( $7^{2} 33^{6}$ inséminations avec $45^{I}$ éjaculats de Taureaux

Coefficient de corrélation de rang $\rho=1, \infty 00$, la courbe calculée d'aprìs

l'équation de fertilité générale.

La concordance est encore très bonne dans la forme transformée (fig. 2)

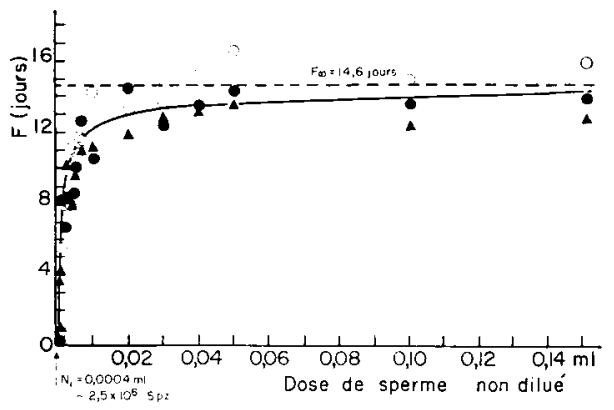

FIG. 3. - Relation entre le nombre de spernatozoüdes inséminés et les résullats de técondation che les Volailles (trois races de Poules différentes), calculée d'après les données expérimentales de TANEJA el Gowe (1962).

Les résultats de fécondation sont indiqués ici par le nombre de jours pendant lesquels la Poule continue encore à pondre des oufs fécondés après l'insémination. Les races présentent de nettes différences de fertilité, si bien que l'étalement peut encore être diminué par l'analyse des données de chaque race prise séparément. Le degré de concordance est mis encore plus nettement en évidence après transformation des données (fig. 4).

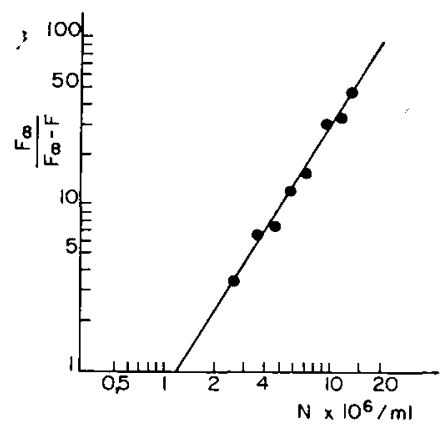

FIG. 2. - Transfornation linéaire des données de la figture 1

(Échelles logarithmiques; coefficient de corrélation linéaire $r=0,98, \mathrm{P}<0,000 \mathrm{I}$ )

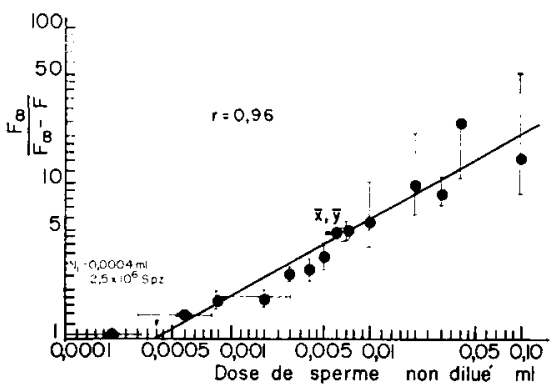

FIG. 4. -- Transformation linéaire des données de la figure 3,calculée sur les moyennes avec erreur-lype des trois races

Dans les doses de sperme les plus faibles, l'erreur est pratiquement entièrement due à la précision de la dose plutôt qu'à la variation de la fertilité, alors qu'avec les fortes doses, les rapports entre ces erreurs s'inversent. (Échelles logarithmiques; coefficient de corrélation linéaire $r=0,96, P^{3}<10^{-8}$ ). 


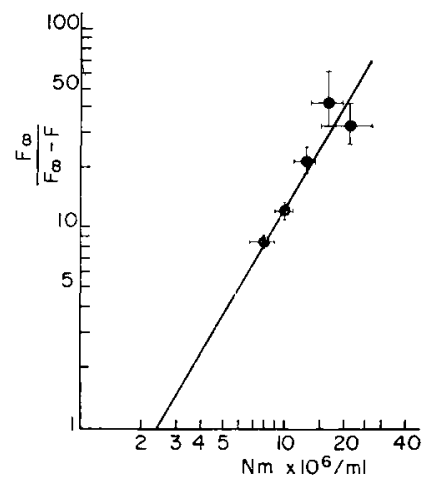

$\mathrm{FI}_{\mathrm{I}}$, 5. - -. Transformation linéaire des données expérimentales de BRANTON, KELIGREN et PATRICK (1953) (8356 inséminations avec 275 éjaculats de Taureaux).

En abscisses ont été portés les produits du nombre total des spermatozoides $Y$ par la motilité estimce à vue d'ocil $m$, l'erreur est alors quatre fois plus forte que dans le cas de $\mathrm{X}$ ci-dessus. La concordance est excellente: coefficient de corrélation linéaire $r=0,9,3, \mathrm{P}<0,000 \mathrm{I}$.

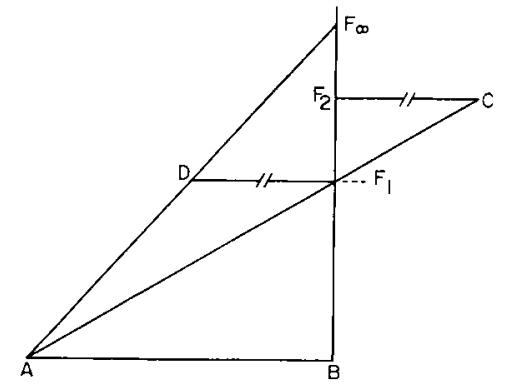

Fic: 6. -- Détermination de Ix a l'aide de la construction de Heyinecker (1925)

sur une base $A B$ dont la longueur correspond à l'unité d'échelle choisie, on trace en $B$ une verticale sur laquelle sont ins. crits dans la mêtme unité, les résultats de fécondation $F_{1}$ et $F_{2}$ correspondant à deux dilutions dans le rapport i à 2 du méme sperme. Par $\mathrm{F}_{\mathrm{t}}$ et $\mathrm{F}_{2}$, on méne deux parallélles à $\mathrm{AB}$. On joint $\mathrm{AF}_{1}$ et prolonge cette droite jusqu'à son intersection C avec la parallèle passant par $\mathrm{F}_{2}$. On porte ensuite, sur la paralléle passant par $\mathrm{F}_{1}$ et vers la gauche à partir de $\mathrm{F}_{1}$, un segment $\mathrm{F}_{1} \mathrm{D}$ égral it $\mathrm{F}_{2} \mathrm{C}$. On trace ensuite la droite $\mathrm{AD}$, son prolongement longe la verticale au point qui représente la meilleure valeur de $\mathrm{F} \infty$ sur l'échelle choisie.

8356 inséminations provenant de 275 éjaculats de taureaux. La transformation faite d'après la théorie, permet d'obtenir $r=0,93, \mathrm{P}<0,00 \mathrm{I}$, alors que le calcul incorrect fait par les auteurs cités a donné un " $r)=0,2 \mathrm{Ig}, \mathrm{P}<0$,or (voir fig. 5).

La pente de la droite $k_{\mathrm{F}} / k_{\mathrm{N}}$ dans le matériel de WILLETT et LARSON (I952) était de I,7 et clans le matériel rassemblé par BRANTON, KELI.GREN et PATRICK (I953) $k_{\mathrm{F}} / k_{\mathrm{x}_{m}}$ fut trouvée égale à $\mathrm{I}, 8$.

Les données expérimentales sur les bozins donnent pour le nombre total de spermatozoïdes inséminés $\mathrm{N}_{i}$ une valeur de l'ordre de $2,0 \times \mathrm{IO}^{6}$ avec un intervalle de variation de $\mathrm{I}, \mathrm{O}$ à $3,0 \times 10^{6}$. Ceci correspond au résultat obtenu pour les volailles dont le $\mathrm{N}_{i}$ pouvait être fixé à $2,5 \times$ Io $^{6}$.

Le peu de matériel sur les porcins laisse suppose que la valeur est légèrement élevée, en tout cas du même ordre de grandeur.

\section{Appréciation de la fertilité d'après les caractéristiques de la motilité et la durée de survie des spermatozoïtes}

L'équation générale de fertilité peut être analysée tant pour la part femelle que pour celle du mâle dans les chances de fécondation.

Pour cela, il faut entreprendre des expériences d'insémination sur une large échelle. Pour apprécier le sperme utilisable à des fins d'insémination, il faut pratiquer, 
une combinaison de paramètres facile à déterminer, qui est exclusivement fonction du sperme. La formule qui convient pour cela peut se déduire ainsi :

Tout d'abord, les spermatozoïdes sont fournis à une certaine distance dans l'espace et dans le temps de $\Delta(p, t)$ de 1'ovule. Celui-ci peut donc être présent ou non au moment où le nombre minimum requis de spermatozoïdes atteint le lieu de rencontre $p$.

La condition minimum pour la réalisation de l'acte de fécondation est donc la présence à l'instant $t$ d'un ovule fécondable à l'endroit $p$, et d'au moins $\mathrm{N}_{i}+\delta \mathrm{N}$ spermatozoïdes fertiles dans le champ entre $\delta p$ et $p$ dont un nombre $d N$ à une distance $\delta(p, t)$ de l'ovule. $\delta \mathrm{N}$ est, en l'occurence, le nombre minimum de spermatozoïdes assurant l'acte de fécondation. Si nous admettons que le mouvement actif est nécessaire pendant la dernière période, $\mathrm{N}$ doit être remplacé par $\mathrm{N} \cdot \bar{v}$, ce qui ne change rien à la forme de la fonction.

L'intégration directe de l'équation donne l'équation exponentielle bien connue :

$$
\mathrm{N}(t)=\mathrm{N}_{0} \cdot e^{-k_{\mathrm{N}} \cdot t} \text {. }
$$

Lorsque dans cette formule $\mathrm{N}(t)=\mathrm{N}_{i}, t$ représentera la durée pendant laquelle le nombre des spermatozoïdes suffisent encore à maintenir une chance de fécondation positive :

$$
t=\frac{\mathrm{I}}{k_{\mathrm{x}}} \cdot \ln \frac{\mathrm{N}_{o}}{\mathrm{~N}_{i}^{-}} \cdot
$$

Cette équation fournit une norme pour la fécondance de la semence, puisque la chance qu'une rencontre féconde avec un ovule apparaissant arbitrairement, se produise, doit s accroître avec la durée pendant laquelle des spermatozoïdes fécondants sont disponibles sur place, c'est-à-dire $\mathrm{F}=f(t)$. Il s'ensuit de (2) que $t$ doit être proportionnel à $l n \mathrm{~F}$. Si nous introduisons le concept période de demi-valeur qui correspond à la réduction de moitié du nombre de spermatozoïdes mobiles $t_{1 / 2}=$ (ln 2)/k, il doit exister une relation linéaire entre le logarithme de la chance de fécondation prévue et $t_{1 / 2}(\mathrm{~N}) \cdot \log \left(\mathrm{N}_{0} / \mathrm{N}_{i}\right)$, lorsque $t_{1 / 2}(\mathrm{~N})=$ période de demi-valeur du nombre des spermatozoïdes fécondants $\mathrm{N}$, et $\mathrm{N}_{0}=$ le nombre des spermatozoïdes fécondants vivants au moment de l'insémination. Si $\mathrm{N}_{i}$ est petit en proportion de $\mathrm{N}_{0}, \log \left(\mathrm{N}_{0} / \mathrm{N}_{i}\right)$ s'approchera de $\log \mathrm{N}_{0}$, parce que $\mathrm{N}_{i}$ a une limite inférieure absolue de I. Si l'on ne dispose pas de données qui permettent une estimation de $\mathrm{N}_{i}$ pour chaque cas distinct, la qualité de la semence par rapport à la fécondance peut être exprimée en tout cas dans un barême logarithmique par $t_{1 / 2}(\mathrm{~N} v) \cdot \log (\mathrm{N} \bar{v})_{0}$, lorsque la motilité est aussi prise en considération, ou bien par $t_{1 / 2}(\mathrm{~N}) \cdot \log \mathrm{N}_{0}$, si la motilité demeure stable, tel est le cas par exemple d'une comparaison de l'influence qu'exercent diverses doses d'un même éjaculat.

Un argument pour l'élimination de $\mathrm{N}_{i}$ existe, si la variance de $\mathrm{N}_{i}$ est relativement petite en proportion de $\mathrm{N}_{0}$, (même si $\mathrm{N}_{i}$ ne répond pas à ce critère). Dans ce cas là $\mathrm{N}_{i}$ pourrait être pris pour une constante de la population.

Il existe une corrélation très poussée entre les deux caractéristiqutes de fertilité :

$$
\begin{aligned}
& \log \mathrm{F}=f\left(t_{1 / 2}(\mathrm{~N} \bar{v}) \cdot \log (\mathrm{N} \bar{v})\right. \\
& \log \mathrm{F}=f\left(t_{1 / 2}(\mathrm{~N}) \cdot \log \mathrm{N}\right)
\end{aligned}
$$


La relation est linéaire pour les logarithmes, si bien que la corrélation est indiquée par:

$$
\begin{array}{cc}
\text { Corr. } \log \left[\left(t_{1 / 2}(\mathrm{~N} \vec{v}) \cdot \log (\mathrm{N} \bar{v})\right]\right. & \text { et } \quad \log \left[\left(t_{1 / 2}(\mathrm{~N}) \cdot \log \mathrm{N}\right)\right] \\
r=0,9 \mathrm{I} & \mathrm{P} \ll \mathrm{IO}^{-6}
\end{array}
$$

D'après cette formule on peut estimer qu'en ne prenant pas en considération la vitesse moyenne des spermatozoïdes on perd près de $I_{5} \mathrm{p}$. Ioo du total de l'information disponible.

Pour vérifier que l'estimation de la fertilité ( 8 ) est utilisable en pratique, les résultats de mesures obtenus avec la méthode photo-électrique de RikMENSPOEI, (I957) appliquée à 40 Taureaux pris arbitrairement, ont été comparés au total des résultats de fécondation obtenus dans la même province (Utrecht) au cours de la même période ( 4 ans). Le résultat de fécondation étant fonction tant de la Vache que du Taureau il est apparu nécessaire d'analyser distinctement les saisons de stabulation et de pacage, car les paramètres de la Vache accusaient, dans l'équation de fertilité générale, sur ce point, différentes valeurs.

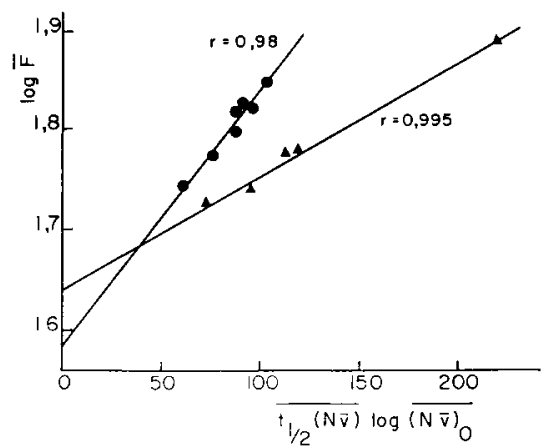

FIG. 7. - Relation entre le logarithme des résultats de fertilité $(\log$ T $)$ et la caracléristique du sperme $t_{1} /{ }_{2}(N \bar{v}) \cdot \log (\mathrm{N} \bar{v})$

Données expérimentales provenant d'éjaculats de Taureaux choisis au hasard sur une période de 4 ans et mises en corrélation avec l'ensemble des résultats d'insémination et tenus dans la même province (Utrecht, i 843 ro inséminations). La pente de la courbe est la constante $k_{F}$ de l'équation générale de fertilité. Elle est fonction de la Vache et présente des valeurs différentes lors des saisons de stabulation et de pacage.

Les unités de l'axe des $x$ sont arbitraires. Les résultats de fécondation sont exprimés en "pourcentages de gestation d'après information ". - pâturage ^ stabulation

La concordance est excellente: comme nous le démontre la figure 7 avec les coefficients de corrélation Corr. $\log \mathrm{F}$ et $t_{1 / 2}(\mathrm{~N} \bar{v}) \cdot \log (\mathrm{N} \bar{v})$.

et

$$
r=0,98, \quad \mathrm{P}<0, \text { oor, pour la saison de pacage, }
$$

$$
r=0,995, \quad \mathrm{P} \sim \mathrm{IO}^{-6} \text { pour la saison de stabulation. }
$$

Ces résultats portent sur I84 3IO inséminations et faisaient intervenir, en moyenne, plus de 13 éjaculats par mois. 


\section{Appréciation de la fécondité du sperme d'après ses caractéristiques biochimiques}

L'énergie consommée par les spermatozoïdes mobiles doit être à tout instant proportionnelle à leur nombre et au carré de leur vitesse moyenne. La vitesse de conversion du substrat $d f / d t$ est donnée par

$$
\frac{d f}{d t}=k \cdot \mathrm{N} \cdot \bar{v}^{2}
$$

Cette équation peut être considérée comme le troisième postulat de la théorie de fertilité globale. Elle permet de mettre les critères de la motilité en rapport avec les paramètres biochimiques. Si $n_{0}$ est le nombre de spermatozö̈des vivants par unité de volume de sperme, $\mathrm{N}$, le nombre de spermatozoïdes vivants inséminés et $f$ la quantité de substrat convertie pendant un temps constant $t$, dans des conditions d'essais normalisées (concentration initiale égale du substrat et conditions de milieu identiques), on peut écrire :

$$
\bar{v}^{2}=\frac{k \cdot f \cdot}{n_{o} \cdot t}
$$

ce qui peut être substitué en (8) et donne :

$$
\log \mathrm{F}=f\left[t_{1 / 2}(\mathrm{~N} \bar{v})\left\{\log \left(\mathrm{N} \sqrt{\frac{t}{n_{0} \cdot t}}\right)+\log \mathrm{K}\right\}\right]
$$

Dans cette formule les constantes sont réunies d'après :

$$
\log \mathrm{K}=\log \sqrt{k}-\log (\mathrm{N} v)_{i}
$$

Dans l'équation $\mathrm{I} 2$, la période de demi-valeur $t_{1 / 2}(\mathrm{~N} v)$ peut être remplacée par un paramètre ne contenant plus la vitesse. Il est plus pratique d'introduire la période de demi-valeur de la consommation de substrat par unité de temps; mais cette technique présente souvent des inconvénients, car les déterminations demandent beaucoup de temps et les quantités de sperme utilisées à cet effet sont plus grandes. On peut aussi utiliser le pourcentage des spermatozoïdes vivants comme critère à la place de la période de demi-valeur, lorsque ce pourcentage est déterminé à un intervalle constant après l'éjaculation au moyen de la coloration à l'éosine des spermatozoïdes morts. $\mathrm{Si} \mathrm{N}_{t}$ est le nombre des spermatozoïdes vivants à un intervalle de temps $t$ constant après l'éjaculation, et $\mathrm{N}_{\mathbf{0}}$ le nombre total des spermatozoïdes, il résulte de (6) que $\log \left(\mathrm{N}_{t} / \mathrm{N}_{0}\right)$ est une fonction linéaire de $t_{1 / 2}(\mathrm{~N})$. Or, d'après les propriétés des fonctions exponentielles, la relation entre les logarithmes des périodes de demi-valeur de diverses quantités fonctionnellement connexes est linéaire et donc l'équivalent de $t_{1 / 2}(\mathrm{~N} v)$ est donné par $\log \log \left(\mathrm{N}_{t} / \mathrm{N}_{0}\right)$.

La fertilité d'un échantillon de sperme peut donc être déduite de la formule suivante :

$$
\log \mathrm{F}=t\left[\log \log \left(\frac{\mathrm{N}_{t}}{\mathrm{~N}_{o}}\right) \cdot \log \left(\mathrm{N} \sqrt{\frac{f}{n_{o} \cdot t}}\right)\right]
$$


Ce rapport a été vérifié sur une analyse des données publiées par Bishop, CAMPBELL, HANCOCK et WALTON (1954) portant sur 3049 inséminations provenant de 63 éjaculats de Taureaux, dont la fructolyse avait été déterminée dans des conditions normalisées (milieu standard, après élimination du fructose du plasme spermatique par dialyse) ; les spermatozoïdes morts avaient été colorés à l'aide d'éosine à un délai constant aprés l'éjaculation. La cortélation obtenue a été de :

$$
r=0,98, \mathrm{P} \sim \mathrm{IO}^{6} \text { (voir fig. } 8 \text { ) }
$$

\section{Le temps de décoloration au bleu de méthylène comme paramètre.}

La détermination du temps pendant lequel une quantité de sperme décolore une quantité constante de bleu de méthylène dans des conditions normalisées, est une méthode largement appliquée. Il doit exister une corrélation directe entre cette caractéristique et la consommation de fructose, étant donné que toutes les deux sont des mesures du métabolisme cellulaire. Cependant, cette corrélation ne peut être linéaire, mais hyperbolique, car le temps nécessaire pour convertir une quantité donnée de substrat est en raison inverse de la quantité de substrat converti pendant un temps constant; le rapport général est indiqué par une équation du type $y=a / x$. Cette condition très élémentaire ne paraît jamais être prise en considération dans la littérature, si bien qu'on ne rencontre que des pseudocorrélations erronées : Corr. $f / t$ et $t_{M}$ (temps de décoloration au bleu de méthylène) qui ont été calculées de façon linéaire. Si au lieu de cela, on avait calculé le coefficient de corrélation de rang $p$, on aurait bien obtenu un résultat correct. Pour pouvoir calculer une corrélation linéaire, le rapport doit être d'abord linéarisé, ce qui peut se faire au choix, soit par voie logarithmique, soit en prenant la réciproque d'une des deux quantités.

Pour les données de Bishop, Campbili, Harcock et Waitox (r954) on pouvait donc calculer : Corr. $\log (f / t)$ et $\log t_{\mathrm{M}}: r=-0,97, \mathrm{P} \ll 0,000 \mathrm{I}$, alors que le calcul erroné Corr. $f / t$ et $t_{\mathrm{M}}$ a donné seulement : $r=-0,79,0,0 \mathrm{I}>\mathrm{P}>0,002$; cette différence est hautement significative (voir fig. g).

Il en résulte que la relation convenable entre la fructolyse et le temps cle décoloration au bleu de méthylène est donnée par l'équation (I3) :

$$
\log \mathrm{F}=f\left[\log \log \left(\frac{\mathrm{N}_{t}}{\mathrm{~N}_{\mathrm{t}}}\right) \cdot \log \left(\mathrm{N} \sqrt{\overline{t_{\mathrm{M}} \cdot n_{o}}}\right)\right]
$$

La vérification du matériel de Bıshor et coll. a donné :

$$
r=0,93, \mathrm{P} \sim \mathrm{IO}^{-5} \text { (voir fig. Io). }
$$

BISHOY et ses collaborateurs calculaient exclusivement le coefficient de pseudocorrélation : Corr. F et $t_{\mathrm{M}}: r=-0,24,0.05>\mathrm{P}$, alors que la véritable corrélation linéaire pour ce rapport simple est : Corr. $\log \mathrm{F}$ et $\log t_{\mathrm{M}}: r=-0,49, \mathrm{P} \sim 0$,or, ce qui est d'ailleurs considérablement en dessous du résultat obtenu avec le critère complet calculé d'après l'équation ( $\mathrm{I}_{4}$ ), comme on peut le prévoir, si les déductions théoriques sont correctes. 


\section{Baisse du pH à l'incubation comme caractéristique de fertilité}

Dans la pratique on se sert parfois aussi de la baisse du $\mathrm{pH}$, pour juger la qualité de la semence. On mesure cette baisse après une incubation de I à 2 heures à température constante (élevée). Mais cette baisse de $\mathrm{pH}$ est non seulement fonction du nombre de spermatozoïdes vivants et de leur activité mais aussi de toute une diver-

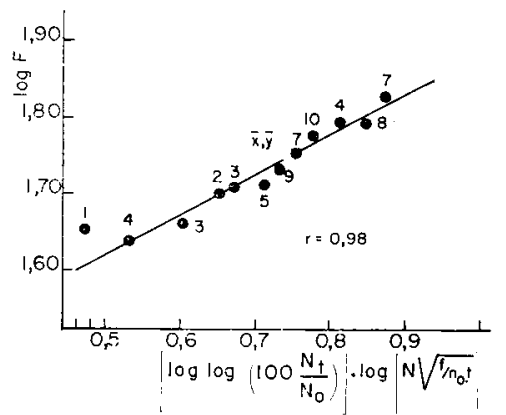

Fir. S. -.- Relation entre le logarithme des résultats de fertilité chez des Bovins (exprimés en " laux de conception ") et la caractéristique de fertilité théorique d'après l'équation (13) avec comme parametres, la tructolvse, le nombre de spermalosoides inséminès el le pourcenlage de spermatosoüdes vivants, déterminés à un monent constant après l'éjuculaition.

A côté de chaque point est indiqué le nombre des éjaculats correspondants ; ceux-ci constituent les facteurs pondéraux des points pour le calcul de la corrélation et la régression. (Calculs effectués sur les données de I3LHOP et coll., 1954).

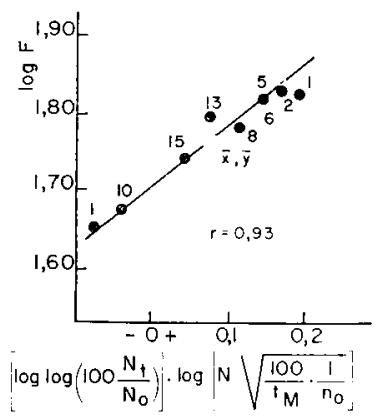

FIC. Io. - Relation entre le logarithme des résultats de fertilité chez les Bovins et la curactéristique de fertilité théorique d'après l'équation (14) anec, comme paramitre, le temps de décoloration du bleu de méthylène. Mêne matériel que pour la fugure 8 .

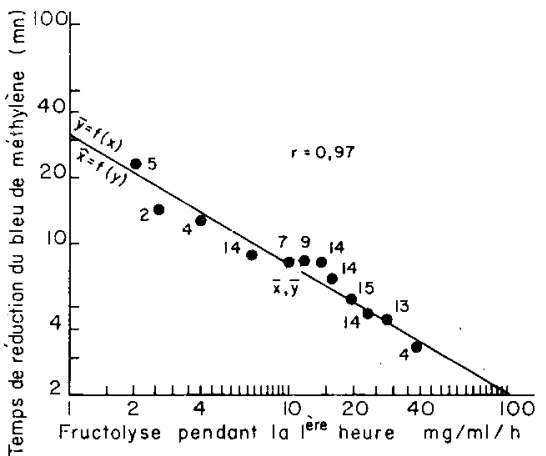

FIG. 9. - Relation entre le temps de décoloration tu bitu de mélhylene et la fructolv'se

(échelle logarithmique pour les $x$ et les $y$ )

A côté de chaque point figure le nombre d'éjaculats par catégorie (calculs effectués sur les mesures de Bishop et coll. I95t)

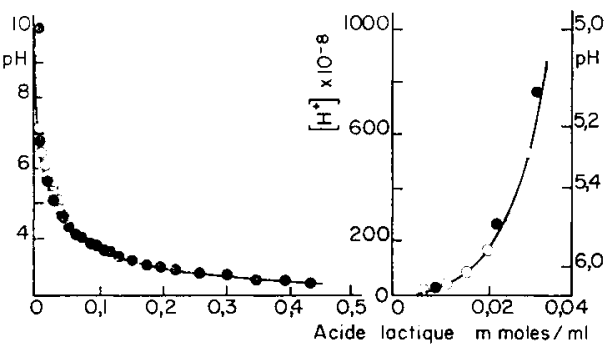

FIG. II. - Relation entre la quantité d'acide lactique et le pHI pour le milieu citrate de sodium-jaune d'out isotonique de RIKMENSPOEL

Ce graphique permet de déterminer la quantité d'acide lactique prołuite par la différence entre deux lectures 
sité de circonstances variables du milieu (plasma séminal), notamment la teneur en fructose, de la force ionique, le $\mathrm{pH}$ initial et son pouvoir tampon; aucun rapport exact ne peut exister entre la baisse du pH initial du sperme et la qualité, même si les facteurs distincts sont causalement déterminés.

I,e protocole de la mesure doit ètre conçu comme suit :

Partant d'un volume de sperme connu, le plasma séminal est éliminé par une centrifugation modérée et les spermatozoïdes sont lavés si nécessaire. Ils sont ensuite resuspendus dans un volume de milieu standard minutieusement mesuré contenant une quantité suffisante de métabolite et raisonnablement tamponné, l'incubation doit alors s'effectuer à l'obscurité en conditions d'anaérobiose.

Le maximum d'informations est obtenu par un enregistrement continu du $\mathrm{pH}$ au cours de l'incubation. On peut ainsi déterminer non seulement la baisse du $\mathrm{pH}$ pendant un espace de temps constant, mais encore la vitesse d'évolution du processus métabolique.

Si les essais sont entrepris de cette façon, on peut établir un rapport sûr entre les résultats observés et les propriétés des spermatozoïdes. La conversion du fructose et du glucose dans des conditions anaérobies donne principalement de l'acide lactique comme produit final.

Le rendement de cette conversion est de 80 à $83 \mathrm{p}$. Ioo pour les spermatozoïdes de Taureatix (Shergin, I937; Rothschild, I962 ; De Groot, I963) et d'environ 43 p. Ioo pour les spermatozoïdes de Béliers (SHERGIN, I937). Si l'on tient compte de ce facteur de rendement, la détermination de l'acide lactique produit permet donc de calculer aussi la quantité totale de fructose converti. Il doit donc exister une relation causale simple entre la quantité de fructose consommée dans des conditions constantes pendant un certain temps et la quantité d'acide lactique produit dans ce même temps. Toutes les équations établies à partir de la consommation de substrat (fructose) comme paramètre, doivent donc pouvoir être converties en équations de la même forme en prenant comme paramètre la production d'acide lactique. Comme ce dernier est dans des conditions anaérobies pratiquement le seul acide produit en quantité perceptible, la baisse totale du pH peut être imputée à l'acide lactique formé, ce qui signifie que la production de l'acide lactique peut être mesurée par la baisse du $\mathrm{pH}$. Cette relation ne peut évidemment pas être rectiligne, même si 1'on remplace l'unité logarithmique du $\mathrm{pH}$ par la concentration correspondante d'ions d'hydrogène, d'une part, parce que l'acide lactique est un acide faible dont le taux d'ionisation change fortement avec la concentration et, d'autre part, parce que le milieu standard à utiliser doit posséder à tout instant certain pouvoir tampon. La relation entre la quantité d'acide lactique et le $\mathrm{pH}$, est pas conséquent curviligne (voir fig. II).

Il faut donc établir un graphique de référence pour le milieu standard utilisé en présence de diverses concentrations d'acide lactique. A partir d'un $\mathrm{pH}$ initial identique, on peut alors déterminer la production d'acide lactique correspondante par une seule lecture du $\mathrm{pH}$ final, après quoi on peut appliquer l'équation (I3) pour l'appréciation de la fertilité.

La méthodologie indiquée ainsi dans son principe est beaucoup plus simple et plus rapide que les déterminations de la consommation du fructose. Les résultats expérimentaux doivent nous indiquer la précision de mesure réalisable. I,e point essentiel est toutefois que la théorie indique comment et dans quelles circonstances 
une baisse du $\mathrm{pH}$ au cours de l'incubation peut en principe servir de paramètre pour la mesure de la fertilité.

\section{Relation entre les nombres de spermatozoïdes morphologiquement anormaux et la fertilité. Relation avec d'autres paramètres}

La relation théorique entre les anomalies morphologiques des spermatozoïdes et la stérilité des cellules afférentes (y compris l'incapacité de développement d'un embryon normal) est donnée par la valeur seuil de $\mathrm{N}_{i}$, dans l'équation de fertilité générale. Elle peut en être séparée pour des usages pratiques par l'introduction d'un facteur $p$ dans les critères d'appréciation selon (8), (9), (I3) ou (I4) représentant le rapport entre le nombre des spermatozoïdes normaux et leur nombre total. Cela veut dire que la relation entre la chance de fécondation et le pourcentage de spermatozoïdes exempts de certaines déformations spécifiques, est linéaire pour $\log \mathrm{F}$ $=f(\log p)$.

L'introduction de ce facteur peut cependant être une affaire délicate, car la relation entre les variations de la forme et la fécondance est, en général, inconnue ; ceci se traduit par les interprétations les plus diverses de ce qui est "normal ", ou " anormal " suivant les observateurs. De cette façon pourrait s'introduire une appréciation subjective et peu sûre qui augmenterait l'imprécision des mesures plus que l'information.

Toutefois, on a pu constater chez le Taureau une relation entre deux formes d'anomalies et d'infertilité à savoir : la présence d'acrosomes isolés dans le sperme, donc de spermatozoïdes qui ont perdu leur acrosome et d'autre part, d'acrosomes persistants, c'est-à-dire qui ne peuvent se détacher et qui ont une forme nettement anormale (TEUNISSEN, I946).

Dans les résultats publiés par BisHop et ses collaborateurs (I954) il est fait mention de 27 éjaculats de Taureaux pour lesquels les pourcentages d'acrosomes séparés des spermatozoïdes ont été déterminés. La relation théoriquement prévue a pu être confirmée par la corrélation

$$
\text { Corr. } \log \mathrm{F} \quad \text { et } \quad \log p: r=0,79,0,000 \mathrm{I}>\mathrm{P} \text {. }
$$

Il a été montré, de surcroît, qu'il existe une corrélation nette entre le pourcentage de spermatozoïdes sans anomalies de tête, et le pourcentage de spermatozoïdes vivants déterminés par la coloration différentielle des spermatozoïdes vivants et morts :

$$
\text { Corr. } \mathrm{N}_{t} / \mathrm{N}_{0} \quad \text { et } \quad p: r=0,660,00 \mathrm{I}>\mathrm{P} \text {. }
$$

Comme le rapport entre les spermatozoïdes vivants et morts à un moment constant après 1'éjaculation est fonctionnellement relié à la période de demi-valeur $t_{1 / 2}(\mathrm{~N})$, cela indique que les anomalies morphologiques s'expriment aussi indirectement dans les paramètres cinétiques.

Des recherches entreprises par Var Rosmalen et VAN DUijn (I963) sur l'incidence des variations de forme et de dimensions des spermatozoïdes sur la résistance de frottement en fluide ont démontré que les déformations se traduisent aussi indirectement dans la vitesse cinétique $\bar{v}$ et par là dans la rapidité de migration direc- 
tement mesurée $N \cdot \bar{v}$. Ces recherches ont révélées que les écarts de la forme hydrodynamique idéale (tête normale) accroissent la résistance au courant d'environ I3 p. Ioo diminuant la vitesse de déplacement à peu près de $5 \mathrm{p}$. Ioo pour une dissipation d'énergie égale. Il est apparu aussi que les variations de la forme et de l'épaisseur de la pièce intermédiaire exercent une incidence encore plus grande.

Étant donné ces interrelations, on peut penser que l'introduction de $p$ dans les normes d'appréciation cinétique n'accroîtra pas l'information dans une proportion aussi forte que celle qui pourraît être suggérée par la corrélation assez élevée $\log \mathrm{F}$ et $\log p: r=0,77$, par lequel donc 59 p. Ioo de la variance sont déjà expliqués. La corrélation avec la fructolyse comme paramètre d'après (I3) fut calculée pour les mêmes 27 éjaculats : elle est de $r=0,78$ et elle fut portée à $r=0,86$ par 1'introduction de $\mathrm{N} \cdot p$ au lieu de $\mathrm{N}$.

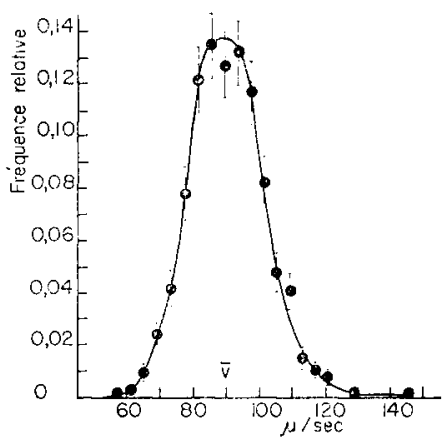

FIG. 12.... Tistribuiton de fréquence normalisée des vitesses movennes des spermatosol̈des provenant de 329 éfacilats de 51 Taureaux différents échantillonnés d'une manière aléatoive.

Cette distribution diffère grandement de la distribution statistique normale, comme le démontrent clairement lés transformations (fig. 1 3 ).

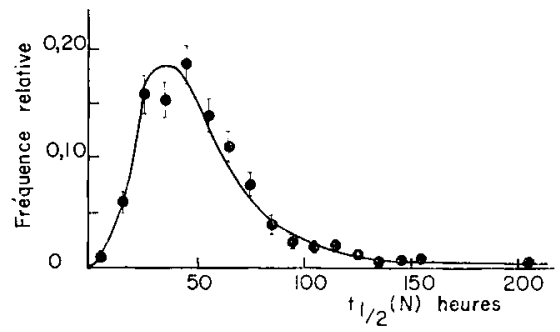

FIG. I 4. - Distribution de fréquence normalisée de la période de demi-valeur des spermatozoilles en mouvement normal de 171 éjaculals de Taureaux au cours de la conservation à $1,9 \div 1,2^{\circ} \mathrm{C}$ (vase de Dewar avec glace).

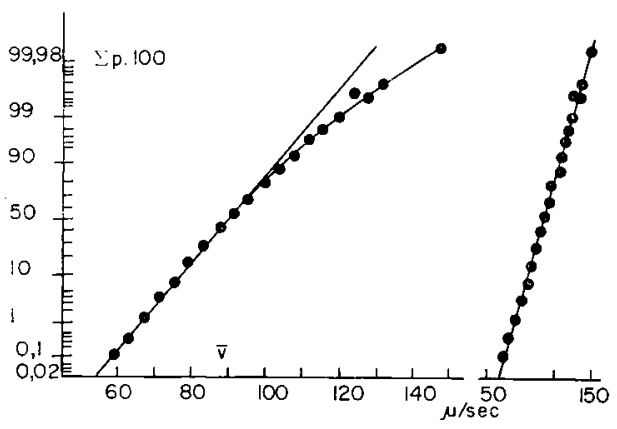

Fis. I 3. -..- Trans/omation des données de la lisure l: sur papier de probabilité normal (a gauche) el logarithmique (à droite)

Elle montre un écart significatif avec la distribution normale, mais une concordance avec la distribution logarithmique.

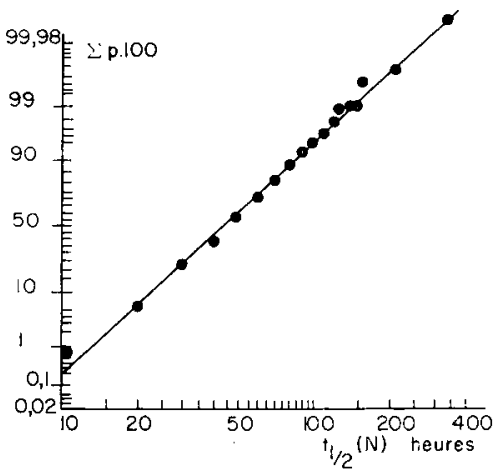

FIG. I5. - Transformation des données de la figure $1 \pm$ sur papier de probabilité logarithnique $x$ échelle logarithmique, $y$ répartition d'apres l'intégrale de probabilité 
Pour ce même matériel, la relation entre le nombre de spermatozoïdes vivants inséminés et Jes résultats de fécondation est significative, elle aussi, si le calcul est fait de façon théoriquement correcte :

$$
\text { Corr. } \log \mathrm{F} \quad \text { et } \log \mathrm{N}: r=0,58,0,02>\mathrm{P}>0, \mathrm{OI} \text {, }
$$

Une comparaison avec la corrélation $\log \mathrm{F} \times \log p$ montre que le pourcentage de cellules privées d'acrosomes pèse plus lourd que le nombre de spermatozoïdes vivants inséminés. Ceci s'explique par la corrélation existant entre $p$ et le rapport spermatozoïdes vivants et morts et aussi avec la période de demi-valeur car celui-ci exerce dans l'équation de fertilité une incidence beaucoup plus grande que dans le nombre.

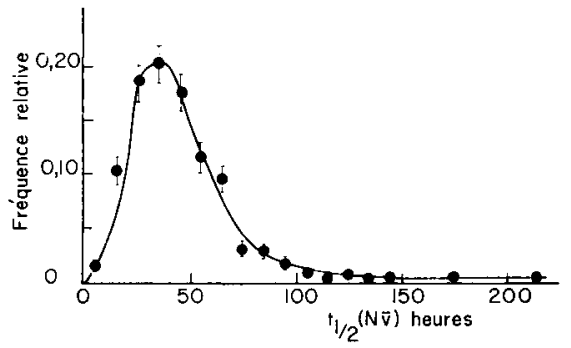

FIG. I6. - Distribution de tréquence nomalisée des périodes de demi-valeur de la rapidité de déplacement $\mathrm{t}_{1 / 2}(N \overline{\mathrm{v}})$ au cours de la conservation dans un récipient de Dewar avec de la glace à $1,9 \pm 1,2^{\circ} \mathrm{C}$ (179 éjaculats)

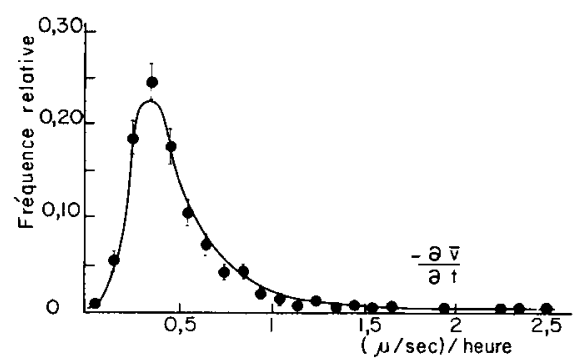

FIG. 18. - Distribution de fréquence normalisée de la rapidité de la baisse de la vitesse avec le temps, - $\partial \overline{\mathrm{v}} / \partial_{t}$ au cours de la conservation d $1,9 \pm 1,2{ }^{\circ} \mathrm{C}$

(186 éjaculats ; mesure de la vitesse moyenne à une température de $37 \pm 0, \mathrm{I}^{\circ} \mathrm{C}$ )

Distribution extrêmement oblique

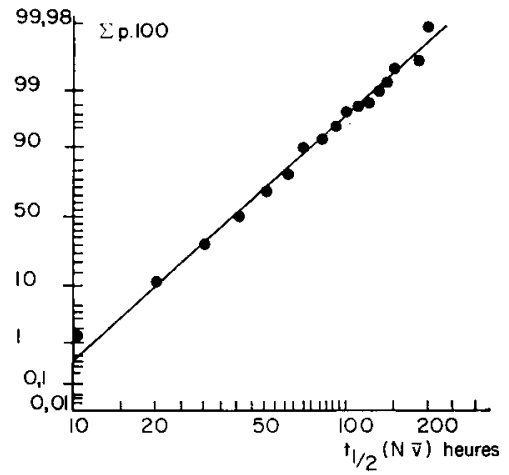

FIG. 17. - Transformation des données de la figure 16 sur papier de probabilité logarithmique

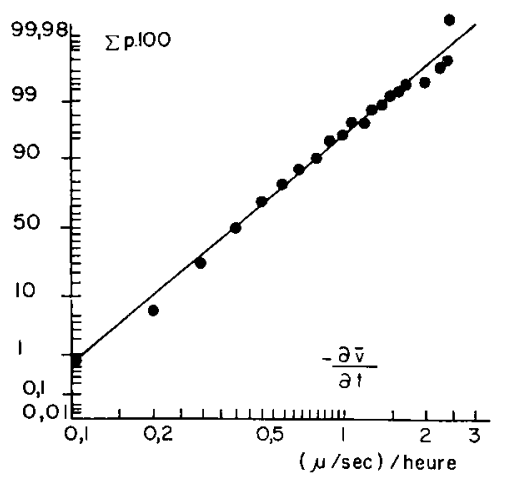

FIG. I9. - Transformation des données de la figure 18 sur papier de probabilité logarilhmique 


\section{III. - LES FONCTIONS DE DISTRIBUTION DES PARAMÈTRES DE FERTILITÉ}

Afin de pouvoir appliquer les statistiques normales de façon justifiée, il est nécessaire de connaître la forme des distributions de fréquence des paramètres à examiner. Cela a été fait pour l'ensemble des paramètres importants pour la théorie de fertilité cinétique à l'aide des données détaillées. Il est apparu alors qu'aucun des paramètres n'était normalement distribué du point de vue statistique.

Cependant, toutes les caractéristiques examinées et consignées dans le tableau ci-dessous pouvaient être définies dans les limites de la véracité expérimentale par une distribution logarithmique qui peut être exprimée sous la forme la plus simple :

$$
y=y_{\mathrm{m}: \mathrm{x}} \cdot \mathrm{e}^{-k^{k}\left(\ln \left(x_{1} x_{m}\right)\right)^{2}}
$$

formule dans laquelle $y=$ fréquence de la valeur du paramètre $x, x_{m}=$ mode de distribution avec la fréquence correspondante $y_{\max } ; k=$ une constante.

Les caractéristiques qui furent jugées distribuées logarithmiquement sont:

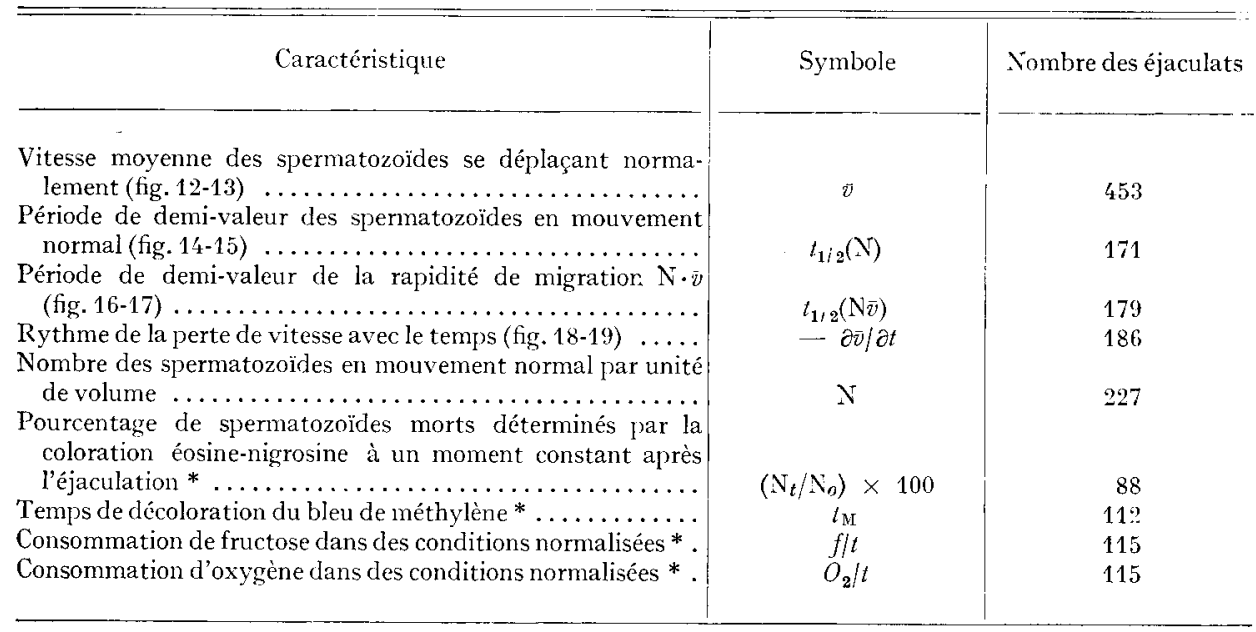

Les distributions de fréquence des caractéristiques marquées de *, furent analysées à partir des donnces de Bishop, Campbell, Hancock et Walton, (195'4).

La fonction de distribution peut être examinée plus simplement par le calcul des fréquences cumulées en les exprimant respectivement sur papier de probabilité normal et logarithmique. Lorsque le résultat sur un des deux ne diffère pas nettement d'une ligne droite, la distribution peut être définie par la distribution de fréquence théorique en cause.

Il en résulte que les méthodes statistiques basées sur la distribution normale, ne peuvent pas être appliquées à l'analyse des résultats de mesure directe. Si 1'on 
y procède quand même, les différences peuvent être jugées, à tort, significatives. Par ailleurs, les moyennes arithmétiques ne sont pas représentatives pour les populations, puisque celles-ci sont intiuencées de manière disproportionnée par des valeurs extrêmej de la distribution oblique. La valeur apparaissant le plus fréquemment, c'est-à-dire le mode de la distribution qui correspond à la moyenne géométrique des observations pett être considérée comme la plus représentative. Elle est définie comme :

$$
\Upsilon^{\prime \prime} x_{1} \cdot x_{2} \cdots x_{n}=\prod_{n}^{n} x=\mathrm{G}_{x}
$$

Lorsque les observations $x$ sont remplacées par le log $x$, la distribution logarithmique devient une distribution statistique normale (distribution de GaUss ou de DE MoIvre), si bien qu'après cette transformation toutes les méthodes statistiques reposant sur la distribution normale, peuvent être appliquées de nouveau, pour autant que la déviation soit indépendante de la moyenne et qu'elle soit du même ordre de grandeur dans les populations à comparer.

$\mathrm{L}_{\mathrm{l}} \mathrm{a}$ nécessité d'utiliser les moyennes géométriques des données ne déroule pas seulement du type logarithme de la distribution des fréquences, mais aussi de l'érolution exponentielle des valeurs paramétriques avec le temps.

C'est seulement quand la dispersion des données est faible que la moyenne arithmétique peut être substituée à la moyenne géométrique dans les conditions limites suivantes:

$$
\lim _{\Delta x \rightarrow 0} \sqrt[n]{\prod_{n} x={ }_{n}^{\mathrm{I}} \Sigma x}
$$

\section{IV. - VARIATION DES PARAMÈTRES}

DE, FERTILITÉ AVEC LES SAISONS

Chez le Tatureau, tous les paramètres relatifs au nombre, à la vitesse moyenne et à la durée de survie des spermatozoïdes semblent subir une variation périodique en rapport avec les saisons. La variation de la moyenne des vitesses moyennes par ćjaculat $\overline{\bar{r}}$ est opposée à celle du nombre de spermatozoïdes en mouvement normal par unité de volume (fig. 20) ceci confirme les résultats obtenus déjà antérieurement sur une corrélation négative entre la vitesse moyenne et le nombre de spermatozoïdes se déplaçant normalement (RIKMENSPOEL, I957: $r=-0,43, \mathrm{P}=0,04$; VAN DUIJN I962: $r=-0,54, \mathrm{P}<0,00 \mathrm{I} ;$ VAN DUIJN, I963: $r-=0,79, \mathrm{P}<0,000 \mathrm{I})$. L'accroissement du coefficient de corrélation et du niveau de signification reflète non seulement l'augmentation des données d'observation, mais aussi la normalisation et la précision de mesures sans cesse améliorées.

On a trouvé également une variation périodique mais en sens opposé entre les périodes de demi-valeur $t_{1 / 2}(\mathrm{~N})$ et $t_{1 / 2}(\mathrm{Nv})$ d'une part, et la perte de vitesse avec le temps $-\partial \bar{v} / \partial t$, d'autre part (fig. $2 \mathrm{I}$ ). Mais ce fait pouvait être prévu, car une forte valeur de la période de demi-valeur signifie une grande longévité, alors qu'une forte valeur de $-\partial \bar{v} / \partial t$, indique une baisse rapide de la motilité. La relation est linéaire pour les 
logarithmes. Les corrélations entre les logarithmes des moyennes géométriques par mois furent trouvées égales à :

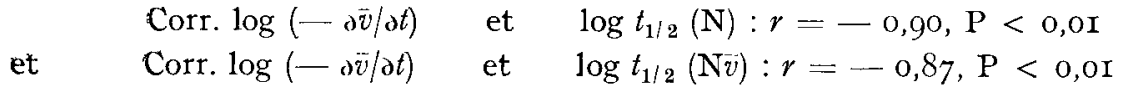

sur une période d'observation de 8 mois avec un échantillonnage arbitraire d'une quarantaine de Taureaux. Sur une période de 16 mois avec un échantillonnage systématique du même nombre de 8 Taureaux le résultat était Corr. $\log (-\mathrm{\partial} \bar{v} / \mathrm{ot}) \times$ $\log t_{1 / 2}(\mathrm{~N} v): r=-0,88, \mathrm{P}<0,000$ or.

Aucune corrélation significative n'a pu être trouvée pour chacune des caractéristiques distinctes avec la fertilité en comparant les moyennes mensuelles, contrairement à ce que l'on obtient, sil'on compare les moyennes géométriques mensuelles de $t_{1 / 2}(\mathrm{~N} \bar{\nu})$ avec la fertilité (voir fig. 22). Les résultats de fécondation sont qualifiés ici de " pourcentage de non-retours" ou de "pourcentage de fécondation d'après les informations ". Le calcul de la concordance s'étaye sur la moyenne de ces deux indications de fertilité, bien que le graphique nous démontre clairement un étalement plus grand pour le " pourcentage de non-retours" que pour le " pourcentage de fécondation d'après information "; la corrélation entre les deux n'est, d'ailleurs pas supérieure à $r=0,79, \mathrm{P}<\mathrm{O}, \mathrm{OI}$, calculée sur toute une année.

Ce résultat ayant trait à une année d'insémination dans la province d'Utrecht, est donc sensiblement moins favorable que celui obtenu par ZWART (I964) sur les données provenant de l'ensemble des Pays-Bas $(r=0,965)$. Il appert, en revanche que la concordance avec les derniers mois de l'exercice (septembre, octobre est plutôt meilleure qu'avec l'ensemble si bien que les résultats de fécondation pour ces mois sont qualifiés - à tort - par ZWART de "invraisemblablement élevés ". I,e degré de concordance entre les résultats de fécondation et la caractéristique de fertilité $t_{1 / 2}(\mathrm{~N} v) \cdot \log (\mathrm{N} \bar{v})$ en fonction de la saison qui résulte déjà du graphique: coefficient de corrélation de rang $\mathrm{p}=\mathrm{I}, 000$; signification de concordance en tendance $\mathrm{P}<0,002$ (test non-paramétrique) ; Corr. $\log \mathrm{F}$ et $t_{1 /}(\mathrm{N} \bar{v}) \cdot \log (\mathrm{N} \bar{v}): r=0,9 \mathrm{I}, \mathrm{P}<0, \mathrm{OI}$.

Cette dernière corrélation ne tient pas compte de la similitude des deux séries 'de valeur pour les deux périodes de l'année, aussi la valeur du test statistique est moindre que précédemment, cependant, le résultat est encore démonstratif. Il est déjà dit plus plus haut que pour cette corrélation sur les données conjuguées dans une période de 4 ans fut trouvé $r=0,98, \mathrm{P}<0,00 \mathrm{I}$, pour la saison de pâturage et $r=0,995, \mathrm{P} \sim \mathrm{IO}^{-6}$ pour la saison de stabulation.

Une comparaison de l'évolution de plusieurs paramètres avec la saison confirme nos conclusions antérieures, à savoir que les caractéristiques de survie $\left(t_{1 / 2}\right.$ et $\left.\delta \bar{v} \delta / t\right)$ sont plus importantes pour l'estimation de la fertilité des spermatozoïdes que la motilité initiale. La corrélation entre une motilité moyenne élevée et une fécondité faible montre qu'une motilité initiale élevée doit être considérée comme une caractéristique défavorable. Au contraire, une fécondité optimale est liée au nombre de spermatozoïdes mobiles et à une longue durée de vie, mais à une vitesse moyenne basse. Ceci est évident dans la corrélation directe entre une grande vitesse initiale et la baisse de vitesse avec le temps: Corr. $\bar{v}_{0}$ (vitesse initiale moyenne) et $(-\partial \bar{v} / \partial t)$ : $r=0,85, \mathrm{P}<\mathrm{IO}^{-12}$. Ce qui entraîne une corrélation négative entre le nombre de spermatozoïdes en mouvement normal et leur vitesse moyenne. 


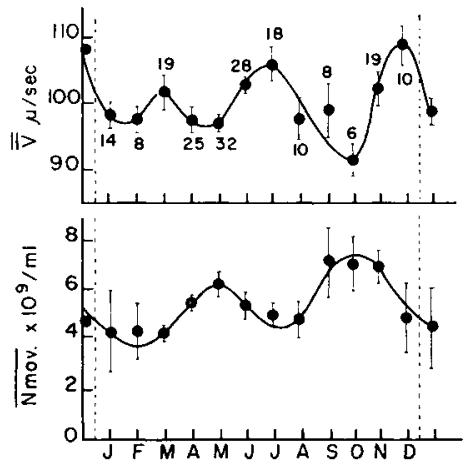

FIG. 20. - Variation saisonnière des vitesses moyennes des spermatozoides dans l'éjaculat de Taureau (en haut) et du nombre nornal de spermatozö̈des en mouvement normal par unité de volume

Le nombre des éjaculats mesurés est indiqué près de chaque point. Évolution nettement opposée entre nombre et vitesse cinétique (39 Taureaux).

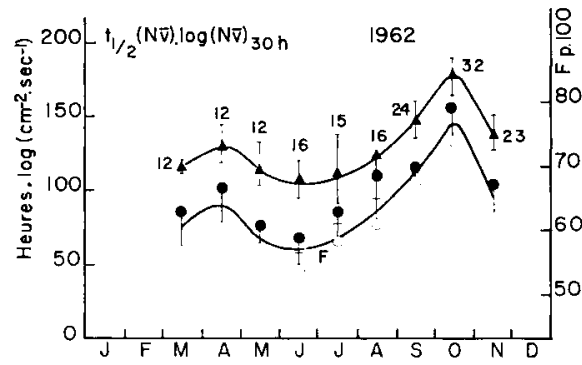

FIG. 22. - Variations saisonnière de la caractéristique du sperme $\mathrm{t}_{1 / 2}\left(N_{i}^{-}\right) \cdot \log \left(N_{\mathrm{r}}\right)$ et des résultats de fécondation

Ces derniers ont été indiqués en is pourcentage de non-retour" (cercles noirs) et en "pourcentage de fécondation d'après information " (cercles blancs); ce dernier indice semble mieux correspondre, en moyenne, à la courbe calculée, que les premiers. Corrélation de tranche entre les observations de la courbe de caractéristique spermatique et les résultats de fécondation I,000, $\mathrm{P}<0,002$.

La valeur numérique de la caractéristique de fertilité est calculée d'après la meilleure estimation de la moyenne du temps entre éjaculations et inséminations ( $3 \circ \mathrm{h})$. Les données d'insémination ont trait à la province d'Utrecht.

^-- Les nombres indiquent le nombre d'éjaculats échantillonés

Taux de conception :

- Pourcentage de non-retours (limite supérieure)

- Pourcentage de fécondation d'aprìs les informations (limite inférieure)

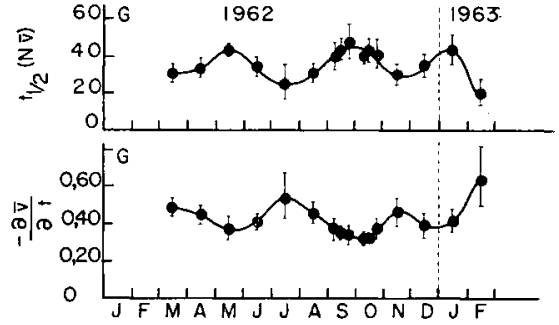

FIG, 2I. -- Variation saisonnière de la période de demi-valeur de la rapidité de migration (en baut) et de la rapidite de baisse de la vitesse (en bas)

Données de 8 Taureaux différents échantillonnés systématiquement

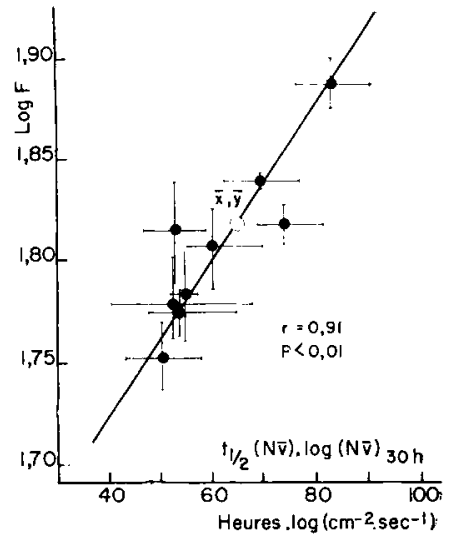

FIG. 23. - Relation entre le logarithme des résultats de fécondation $\log \mathrm{F}$ el $\mathrm{t}_{1 / 2}(N \overline{\mathrm{v}}) \cdot \log (N \cdot \overline{\mathrm{v}})$, d'après les données de la figure 22

(Voir aussizla figure 7) 
La complexité des données démontre que la concordance entre les résultatde fécondation et les caractéristiques du sperme sur toute la saison s'obtient exclus sivement avec l'expression intégrale : $t_{1 / 2}(\mathrm{~N} v) \cdot \log (\mathrm{N} v)$ et non pas avec des caractères distincts. Or, l'équation de fertilité a permis pour la première fois de trouver une quantité caractéristique pour l'appréciation du sperme répondant à la condition rigoureuse de ERB et WALDO (1952) ainsi conçue : tout système d'appréciation spermatique ne peut être considéré comme utilisable que lorsque 1es corrélations aver la fertilité sont maintenues pendant toutes les saisons.

\section{V. - APPLICATION DE LA THÉORIE DE FERTILITÉ POUR L'APPRÉCIATION DU SPERME DANS LA PRATIQUE}

Pour juger du sperme sur la base des caractéristiques cinétiques et de la duŕe de survie, la théorie nécessite donc la détermination de la rapidité de migracion $\mathrm{N} \cdot \vec{v}$ et sa période de demi-valeur. Comme le nombre $\mathrm{N}$ et la vitesse moyenne $v$ se présentent toujours comme un produit et non pas isolément, il suffit de déterminer directement ce produit. $\mathrm{N} \cdot \tau^{\prime}$ ' est, en effet, directement proportionnel au nombre des spermatozoïdes passant par unité de temps et par un point d'observation fixe dans des conditions déterminées. Ceci peut être déterminé de façons diverses et spécialement en appliquant la méthode photo-électrique de Rikmesspof, dont nous avons déjà parlé dans l'introduction de cet article.

Dans la pratique, on peut utiliser une méthode de mesure visuelle, simple ayant, en principe, la même précision. Il faut un hémocytomètre profond de 40 microns (s'il est moins profond, le mouvement est influencé par la proximité trop grande des parois, s'il l'est plus, la profondeur de champ du système optique est trop faible) un microscope avec un objectif $x$ Io et un oculaire x 25 on x 30 (type de Ramsden, ou Kellner) dans lequel est aménagé un filtre de gélatine vert clair. Au milieu de ce filtre, se trouve un petit trou d'un diamètre tel que la reproduction agrandie d'une tête de spermatozoïde s'y adapte justement. Pour les spermatozoïdes de Taureaux, de Verrats, de Béliers et de Chiens, on peut retenir I/ Io mm et pour les spermatozoïdes des humains $\mathrm{r} / 20 \mathrm{~mm}$.

Sous le condenseur du microscope est placé un diaphragme central pour éclairage à fond noir (avec un microscope à contraste de phase, on obtient un éclairage à fond noir en plaçant simplement un diaphragme annulaire du condenseur destiné à un objectif plus fort, ainsi l'anneau ne peut plus être reproduit dans l'objectif). L,e microscope doit être équipé d'une platine chauffante réglée à une température contante ou bien il doit être placé dans une étuve à température constante (tolérance $\left.\pm I^{\circ} \mathrm{C}\right)$. Enfin, on a besoin d'un petit mécanisme compteur et d'une sonnerie d'appel.

Comme les spermatozoïdes sont très sensibles à l'incidence de la lumière (NORMAN et Goldberg, I959; VAN DUIJN, I96I), en particulier dans la zone des longueurs d'ondes inférieures à $500 \mathrm{~m} \mu$ (NORMAX et GOLDBERG, I96I ; NORMAN, GOLDBERG et Porterfieis, I962), un filtre protecteur est placé sous le condenseur contre la lumière. Le filtre Ilford $n^{\circ} 808$, absorbant fortement de $555 \mathrm{~m} \mu$ jusqu'à ultra violet s'est révélé très efficace pour cela. Grâce au filtre perforé vert clair placé dans l'oculaire, l'effet visuel devient un fond noir, dans lequel les spermatozoïdes se trouvant 
à l'extérieur de l'endroit de mesure apparaissent vert foncé, alors que ceux qui passent devant le point de mesure s'éclairent soudainement en orange vif.

Le sperme à mesurer doit être dilué avec un milieu standard suffisamment clair ; mais ces exigences ne sont pas aussi rigoureuses que pour la méthode photo-électrique comportant l'utilisation du milieu de RIKMENSPOEI (I957) avec du jaune d'œuf ultracentrifugé.

La présence de jaune d'œuf dans le médium est d'une importance cruciale.

La dilution doit être effectuée correctement à l'aide de micropipettes de telle sorte que les passages puissent être comptés. Une dilution de 50 à Ioo fois donne, en général, đe bons résultats. Elle doit avoir lieu avec les précautions usuelles pour éviter des chocs thermiques ou autres.

Pour chaque mesure à effectuer, on affiche un temps constant sur la sonnerie d'appel (5 minutes au minimum) et le nombre de spermatozoïdes passant dans ce laps de temps sur le point de mesure est compté. Trois mesures successives sont effectuées par essai, pour lesquelles on prend chaque fois une goutte fraîche de sperme dilué. Cette opération est répétée au bout de quelque temps, pendant lequel le sperme a été conservé dans des conditions constantes. Si l'on veut terminer rapidement l'examen d'un éjaculat, il convient de conserver le sperme dans l'obscurité à la tempéra-

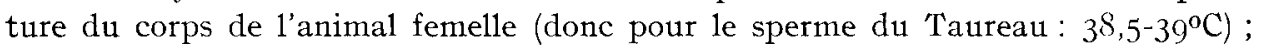
la deuxième série d'observations peut commencer alors au bout de quelques heures déjà. S'il faut, par contre, examiner un grand nombre d'éjaculats que l'on ne va pas utiliser le jour même pour insémination, la conservation peut se faire à une température basse. La deuxième série de mesures peut avoir lieu plus tard. Il est nécessaire de bien définir le protocole d'observation, car les temps de demi-valeur utile dépendent des conditions de conservation.

On procède comme suit :

Doivent être notés : le moment de l'éjaculation et les moments de chaque mesure. Prendre $t_{o}$ comme moment de l'éjaculation (zéro de l'échelle horaire) et inscrire les résultats de mesure obtenus $(\mathrm{N} \bar{v})$ en fonction du temps $t$ sur du papier semi-logarithmique (ordonnées logarithmiques, abscisses linéaires). On obtient donc deux groupes de 3 points. Former le triangle que relient les trois points d'un groupe et construire son centre de gravité (point de croisement des médianes). Relier les centres de gravités des deux triangles par une droite qui est prolongée jusqu'à l'axe $y$. Ce point d'intersection détermine la valeur de $(\mathrm{N} \bar{v})$ au moment de l'éjaculation. Diviser cette valeur par 2, lire le résultat sur l'axe $y$, la valeur correspondante de $t$ sur l'axe $x$ indique alors la période de demi-valeur. La fertilité relative du sperme est exprimée par le produit de $t_{1 / 2}(\mathrm{~N} v)$ avec $\log \left(\mathrm{N}^{-}\right)_{t=0}$. On utilise à cette fin la valeur réelle de $\mathrm{N} v$ calculée en multipliant les passages comptés par le facteur de dilution et la constante de l'appareil. (voir fig. 23.)

Pour estimer les résultats de fécondations probables, il faut déterminer la valeur de $t_{1 / 2}(\mathrm{~N} \bar{v}) \cdot \log (\mathrm{N} \bar{v})$ au moment de l'insémination. Cette valeur peut également être déterminée directement sur le graphique par extrapolation de la ligne droite et par

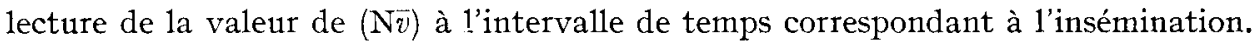
Le résultat doit être multiplié par le nombre de $\mathrm{ml}$ de semence diluée inséminé et divisé par le facteur de dilution. Ie logarithme de la chance de fécondation $\mathrm{F}$ est, par conséquent, une fonction rectiligne du produit $t_{1 / 2}(\mathrm{~N} \bar{v}) \cdot \log (\mathrm{N} \bar{v})$.

Une méthode permettant de déterminer la caractéristique plus restreinte 
$t_{1 / 2}(\mathrm{~N}) \cdot \log \mathrm{N}$ contenant environ I5 p. roo d'information en moins, consiste à réaliser une coloration des spermatozoïdes vivants et morts à deux moments déterminés après l'éjaculation. Èn inscrivant de nouveau les résultats sur du papier semi-logarithmique, on peut faire la lecture tant de $N$ que $t_{1 / 2}(N)$ à tout moment. Cette méthode présente 1'avantage de permettre des mesures différées; en temps de grande presse, il suffit de faire les préparations de frottis et de noter les temps respectifs, après quoi les comptages peuvent être effectués à un moment plus tranquille.

D'autre part, il est important de tenir compte du délai entre l'éjaculation, les mesures de contrôle et le moment de l'insémination ; on a pu mettre en évidence une corrélation négative et significative entre les résultats de fécondation et le nombre des inséminations par éjaculat. Ceci peut s'expliquer par le fait que pour un éjaculat, l'âge moyen de la semence augmente avec le 'nombre des inséminations, en effet, avec ce dernier, augmente le temps passé à inséminer et le temps de transport. Dans le matériel publié par Bishop et ses collaborateurs (I954) où les nombres d'inséminations par éjaculat variaient de moins de ıo à plus de 200 , on a vérifié qu'une relation existait entre le nombre des inséminations et la qualité, déjà connue par expérience, des Taureaux utilisés. La corrélation entre la fécondance $(y)$ et le nombre d'inséminations par éjaculat $(x)$ est :

$$
r=-0,70, \mathrm{P}<0, \mathrm{or}
$$

avec une équation de régression répondant à la formule :

$$
y=-0, \mathrm{I} x+63,3
$$

Cette régression est valable pour un nombre de 5 à 220 inséminations par éjaculat : bien que d'après sa pente $(O, I)$ son incidence ne soit pas très grande, elle contribue cependant de façon nette à l'étalement total des résultats observés.

Si les valeurs mesurées sont ramenées à celles existant au moment de l'éjaculation, par application de la méthode graphique qui a été décrite, cette cause d'erreur peut être complètement éliminée.

Pour l'ensemble des données de Bishop et al., les nombres d'inséminations à partir de chaque éjaculat sont répartis au hasard dans les différentes classes ; la corrélation entre le nombre d'inséminations par éjaculat et la fécondité a donc seulement pour conséquence une augmentation de la dispersion sans pour autant introduire une erreur systématique.

Reçu pour publication en février 1965.

\section{SUMMARY}

\section{RELATIONSHIP BETWEEN CHARACTERISTICS OF SPERMATOZOA AND FERTILITY}

r. The relationship between number, motility and longevity of spermatozoa and the probability of fertilization is shown to be given by the general fertility equation :

$$
\mathrm{F}=\mathrm{F}_{\infty}\left\{\mathrm{I}-\exp \left[-\frac{k_{\mathbf{F}}}{k_{\mathrm{N} v}} \cdot \ln \frac{(\mathrm{N} \bar{v})_{o}}{(\mathrm{~N} \bar{v})_{i}}\right]\right\}
$$

where $\mathrm{N}=$ number of normally moving spermatozoa with mean velocity $\bar{v} ;(\mathrm{N} \bar{v})_{0}=$ value of the product $N \cdot \bar{v}$ (migration rate) at the time of ejaculation during natural coitus, or of insemination in 
artificial breeding; $(\mathrm{N} y)_{i}=$ threshold value required to make the next spermatozoön shift the probability of fertilization from $\mathrm{F}=0$ to $\mathrm{F}>0 ; \mathrm{F} \infty=$ highest probability of fertilization that can be achieved in a given population; $k_{\mathrm{F}}=$ rate constant of the fertilization process, governed by the female ; $k_{N v}=$ rate constant of deterioration of the spermatozoa $=0.693 / t_{1 / 2}(\mathrm{~N} \widetilde{v}), t_{1 / 2}(\mathrm{~N} v)$ being the half-life period of the migration rate $\mathrm{N} \cdot \overrightarrow{\mathrm{v}}$. ship :

2. The quality of an ejaculate with respect to fertility may be measured by the linear relation-

$$
\log \mathrm{F}=f\left[t_{1 / 2}(\mathrm{~N} \bar{v}) \cdot \log (\mathrm{N} \bar{v})\right]
$$

or, if variations in velocity are neglected, by :

$$
\log \mathrm{F}=f\left[t_{1 / 2}(\mathrm{~N}) \cdot \log \mathrm{N}\right]
$$

The second fertility index contains about i $5 \mathrm{p}$. Ioo less information than the former.

3. The half-life period $t_{1 / 2}(\mathrm{~N} \bar{v})$ may be substituted by a function of the live/total ratio $\mathrm{N}_{t} / \mathrm{N}_{0}$, determined at a constant time after ejaculation, whereas $N \bar{v}$ may be substituted by a function of either fructolysis or methylene blue reduction time $t_{\mathbf{M}}$. With $\mathrm{N}=$ number of living spermatozoa inseminated and $f /\left(n_{0} \cdot t\right)=$ fructose consumed per spermatozoön per unit time under standardised conditions, it is shown that the corresponding relationships are :

or

$$
\begin{aligned}
& \log \mathrm{F}=f\left[\log \log \left(\frac{\mathrm{N}_{t}}{\mathrm{~N}_{o}}\right) \cdot \log \left(\mathrm{N} \sqrt{\frac{f}{n_{o} \cdot t}}\right)\right] \\
& \log \mathrm{F}=f\left[\log \log \left(\frac{\mathrm{N}_{t}}{\mathrm{~N}_{o}}\right) \cdot \log \left(\mathrm{N} \sqrt{\frac{\mathrm{I}}{\tau_{\mathrm{M}} \cdot n_{0}}}\right)\right]
\end{aligned}
$$

( $n_{\mathrm{o}}=$ sperm density in the fructolysis or methylene blue reduction measurements).

4. All relationships mentioned have been checked on experimental data and were found to be in complete agreement, correlation coefficients in the linearized forms of equations (2) - (5) being in the range of $r=0.93-0.995$.

5. It is explained how decrease of $\mathrm{pH}$ under standardised conditions, including separation of the spermatozoa from the seminal plasma and resuspending them in a suitable standard medium with glycolysable substrate, can also be used as a quality index for the fertility of spermatozoa.

6. The effect of morphological abnormalities is discussed and shown to be accounted for in the value of $(\mathrm{N} \bar{z})_{i}$ of the general fertility equation (I) ; if required it can be introduced as a separate parameter in the fertility indices after (2) - (5), but owing to intercorrelations with the other parameters the gain is little and there is a risk of introducing more noise than true information into the relationship.

7. The frequency distributions of all spermatozoan parameters investigated are found to be logarithmic. Consequences for statistical analysis are discussed.

8. Seasonal variations have also been studied. In the month where fertility is highest, the average mean velocity of bull spermatozoa is lowest, whereas average numbers of normally moving sperms and half-life periods of numbers and of migration rates are highest. None of these parameters follows the same course as fertility over the whole year, but the kinetic fertility criterion (I) shows perfect agreement.

9. A simple experimental procedure is described for determining this criterion, consisting of counting the number of sperms passing by a fixed spot in the dark-field microscope, indicated by a hole in a green filter screen mounted on the diaphragm of $a \times 3$ o or $\times 25$ corrected Ramsden type eye-piece. Between the light source and the microscope an orange filter (Ilford No. 808 or equivalent) is placed to protect the spermatozoa from light during the measuring period of 5 minutes. A series of measurements is evaluated graphically.

Io. A significant negative correlation $(r=-0.70$, o.o I $>\mathrm{P})$ has been found between fertilization results and the number of inseminations per ejaculate (in the same range of sperm densities used for insemination), which is explained by the increasing effect of ageing during the period of time needed for performing ever more inseminations. 


\section{RÉFÉRENCES BIBIIOGRAPHIQUES}

Bishop M. W. IT., Campbell R. C., Hancock J. L., Walton A., r954. Appendice to : Semen characteristics and fertility in the bull. J. Agric. $\mathrm{Sci}_{\mathrm{i}}, \mathbf{4 4}, 227^{-2}+8$.

BrantoN C., Kellorex H. C., PATRICK T. Ii., 1953. The importance of numbers of spernatozoa in relation to semen quality and fertility of bulls. J. Iain Sci.. 36, 1301-1307.

Dujs C. Van Jr., I96r. Effects of light and optical sensitization by acridine-orange on living bull spermatozoa. Nature, $\mathbf{1 8 9}, 76-78$.

Dusj C. Van Jr., 196r. Photodynamic effects of vital staining with diazine green (Janus green) on living bull spermatozoa. Exp. Cell. Res., 25, 120-1 30 .

Duin C. Van Jr., 1961. Effects of light and photosensitizat ion by some vital stains and fluorochromes on living bull spermatozoa. Proc. IVh Int. Congr. Inint. Reprot.. Den Hang 5-9 june 1961, 2, $249^{-2} 55^{2}$

DuJj C. Van Jr., 1962. Velocity characteristics and numbers of bull spermatozoa in relation to ageing, determined by photo-electric methods. J. Reprod. Fertil., 4, $277-290$.

DuIJ C. Van Jr., I963. (Bevruchtend vermogen van spermatozoa in verband met hun beweeglijkheidskenmerken en overlevingsduur. I. Kinetische theorie van de bevruchtingskans). Pouvoir fécondant des spermatozoìdes en relation avec leurs caractéristiques de motilité et leur durée de survie. I. Théorie cinétique du pouvoir fécondant. Rapport No $B 48$ van het Institum voor Veeteelthundig Ondersoek "Schoonoord".

Duın C. Van Jr., 196.4. (Ilet verband tussen aantal, beweeglijkheid en overlevingsduur van spermatozoa en de bevruchtincrskans.) Relation entre le nombre, la notilité et la durée de survie des spermatozoïdes et le pouvoir fécondant. Ned. Tijdschr. Geneesk., 108, т 1 16-г I23.

Dujn C. Van Jr., r964. Kinetics of fertility of semen. Proc. Vth Internat. Congr. Anim. Reprod., Trento.

DujJs C. Van Jr., I964. A rational method for estimating fertility of spermatozoa in ritro. Proc. Vith Internat. Congr. Inim. Reprod., Trento.

Duijn C. Van, Jr., I g64. Parameters of spennatozoan movement in relation to season, of bulls in the Netherlands. Proc. Vih Internal. Congr. Anim. Reprod., Trento.

DuIJn C. Van Jr., I964. The frequency distributions of velocity characteristics and biochemical parameters of bull spermatozoa. Proc. Vh Internat. Congr. Animt. Reprod.. Trento.

Dujun C. Van Jr., Rikmenspoel R., ig6o. The mean velocity and velocity distributions of normal bull spermatozoa at different hydrogen ion concentrations, derived from photo-elect ric measurements. J. Agric. Sci., 54, 300-309.

ERB E. R., WaLdo D. R., 1952. Seasonal changes in fertility of dairy bulls in Northwestern Washington. J. Dairy Sci., 35, $245^{-249}$.

Groot B. (de), ro63. (De invloed van de pli op de fructolyse en de methyleenlblauw-reductie van stierespermiën.) Influence du $\mathrm{pH}$ sur la fructolyse et la réduction du bleu de méthylène dans le sperme du Taureau. Tijdschr. Diergeneesk., 88, 489-496.

Hancock J. L., Hover G. J. R., 1961. The effect of semen volume and number of spermatozoa on the fertility of intra-uterine inseminations of Pigs. Anim. Prod., 3, 153-16r.

Heydecker W. A., 1925. Résolution rapide de quelques problemes usuels de sensitométrie. Sci. Ind. Phot., 5, 2 I M.

Norman Ch., Goldbers; 1:., I959. Leffect of liwht on motility, life-span, and respiration of bovine spermatozoa. Science, 130, 624.

Norman Ch., Goldber; H., i961. The effect of visible light on spermatozoa. J. Reprod. Ferill., 2, 5II.

Norman Ch., Goldberc; E., Porterfieid I. D., ig62. The effect of visible radiation on the functional life-span of mammalian and avian spermatozoa. Exp. Cell Res., 28, 69-84.

Rrkuenspoer. R., 1957. An optically clear egg-yolk diluent for bull spermatozoa. Experientia, 13, i2+

RikMEnspoeI. R., I957. "Photoalectric: and cinematographic measurements of the "motility " of bull sperm cells. "Dissertatie Utrecht.

Rikmenspoel, R., ig6o. Measurements of the " motility " of bull sperm cells under various conditicns. $J$. Agric. Sci., 54, 399-709.

Rikmenspoel R., VAN Dujun C., Jr., ig6o. (Foto-electrisch en cinematografisch onderzoek van de beweeglijkheid van stierenspermatozoa. II. Foto-electrische metingen van enige eigenschappen van stierenspermatozoa.) Étude photo-électrique et cinématographique de la motilité des spermatozoïdes de Taureau. II. Mesure photo-électrique de quelques propriétés des spermatozoïdes de Taureau. Tijdschr. Diergeneesk., 85, 1002-1014.

Rosmalen W. Van, VAN DUIJN (.., Jr., I963. (D) invloed van vorm en afmetingen op de stromingsweerstand van spermatozoa.) Influence de la forme et des dimensions sur la résistance de frottement en fluide des spermatozoides. Rapport No $B 44$ van het Instillut voor Veeteeltkundig Onderzoek "Schoonoord", Zeist.

RothsciIlld Lord, I962. Anaerobic heat production and fructolysis of bull spermatozoa at different temperatures. J.Exp. Biol., 39, $387-394$.

Suergin N. P., 1937. Probl. Zizoln. no 12, 126. (Anim. Breed. Absir., 7, 18.) 
TANkJA (i. C., Gowe K. S., 1962. Jiffects of varying doses of undiluted semen on fertility and hatchability in the domestic fowl. J.Reprod. Lertil., 4, I6I-1 74 .

Teunissen G. H. R., 1946. (Een afwijking van het acrosoon bij de spernatozoiden van een stier.) Anomalies de l'acrosome des spermatozoïdes de Taureau. Tijdschr. Diergeneesk., 71, 202-303.

Witietr E. L., Larsox G. L., I952. Fertility of bull semen as influenced by dilution level, antibiotics, spermatozoan numbers and the interaction of these factors. J. Dairy Sci., 35, 899-905.

ZWART Th., Ig64. (Het grebruik bij de K.i.-verenigingen van het "nietterugkomerspercentage "als maat voor de bevruchtingsstoestand.) Utilisation du pourcentagre de non-retour comme mesure de la fertilité dans les conpératives d'insémination artificielle. Veeleelt en Zlivelberichlen, 7, I I I-I I 5. 